\title{
ARTICLE
}

Maternal and pediatric nutrition

\section{Socio-economic patterning of food consumption and dietary diversity among Indian children: evidence from NFHS-4}

\author{
Sutapa Agrawal ${ }^{1}$ Rockli Kim $\mathbb{D}^{2} \cdot$ Jewel Gausman ${ }^{3} \cdot$ Smriti Sharma $^{4} \cdot$ Rajan Sankar $^{5} \cdot$ William Joe $^{6} \cdot$ \\ S. v. Subramanian ${ }^{2,7}$
}

Received: 7 August 2018 / Revised: 19 January 2019 / Accepted: 30 January 2019 / Published online: 26 February 2019

(c) The Author(s) 2019. This article is published with open access

\begin{abstract}
Background/Objectives Most interventions to foster child growth and development in India focus on improving food quality and quantity. We aimed to assess the pattern in food consumption and dietary diversity by socioeconomic status (SES) among Indian children.

Subjects/Methods The most recent nationally representative, cross-sectional data from the National Family Health Survey (NFHS-4, 2015-16) was used for analysis of 73,852-74,038 children aged 6-23 months. Consumption of 21 food items, seven food groups, and adequately diversified dietary intake (ADDI) was collected through mother's 24-h dietary recall. Logistic regression models were conducted to assess the association between household wealth and maternal education with food consumption and ADDI, after controlling for covariates.

Results Overall, the mean dietary diversity score was low (2.26; 95\% CI:2.24-2.27) and the prevalence of ADDI was only $23 \%$. Both household wealth and maternal education were significantly associated with ADDI (OR:1.28; 95\% CI:1.18-1.38 and OR:1.75; 95\% CI:1.63-1.90, respectively), but the SES gradient was not particularly strong. Furthermore, the associations between SES and consumption of individual food items and food groups were not consistent. Maternal education was more strongly associated with consumption of essential food items and all food groups, but household wealth was found to have significant influence on intake of dairy group only.

Conclusions Interventions designed to improve food consumption and diversified dietary intake among Indian children need to be universal in their targeting given the overall high prevalence of inadequate dietary diversity and the relatively small differentials by SES.
\end{abstract}

Supplementary information The online version of this article (https:// doi.org/10.1038/s41430-019-0406-0) contains supplementary material, which is available to authorized users.

S. V. Subramanian

svsubram@hsph.harvard.edu

Tata Trusts, New Delhi, India

2 Harvard Center for Population and Development Studies, Harvard University, Cambridge, MA, USA

3 Department of Global Health and Population, Harvard T.H Chan School of Public Health, Boston, MA, USA

4 Tata Trusts, Mumbai, India

5 The India Nutrition Initiative (TINI), Tata Trusts, New Delhi, India

6 Institute of Economic Growth (IEG), Delhi, India

7 Department of Social and Behavioral Sciences, Harvard T.H. Chan School of Public Health, Boston, MA, USA

\section{Introduction}

Food, like air and water, is a must for survival and the value of diversified diet for essential nutrients has long been recognized [1]. Adequately diversified diet, in terms of amount and composition, is critical for optimal growth, development, and long-term health outcomes in children [2]. Most previous research on child nutrition around the world focused on anthropometric failures rather than dietary diversity [1, 3-5]. While dietary diversity and anthropometric failures are inter-related, examining the distribution of dietary diversity and food deprivation across population groups is critical given that most interventions to improve child growth focus on food quality and quantity $[6,7]$.

In India, despite all the advances in health and development [8], the burden of child under nutrition and micronutrient deficiency remains high $[9,10]$. Around $70 \%$ of the children suffer from inadequate dietary intake and $40 \%$ 
remain stunted [11]. While significant progress has been made in improving food production and sufficiency over the last 50 years [12], most rural populations and communities continue to face uncertainty in food security. In aggregate, over onefifth of India's population still suffers from chronic hunger and India ranks 100th among 119 countries in the 2017 Global Hunger Index [13]. A number of organizations have advocated for dietary diversity strategies to tackle the burden of micronutrient malnutrition among children in India [14]. Apart from the existing schemes aimed at reduction in the levels of malnutrition among women and children, the Government of India has recently set up National Nutrition Mission or Poshan Abhiyaan [15] for improving the nutritional status of children, pregnant women, and lactating mothers. The mission aims to prevent and reduce prevalence of stunting among children ( $0-6$ years) in the country by $6 \%$ and undernutrition (underweight) by $6 \%$ [16]. The Mission also emphasizes dietary diversification among the 15 key nutrition strategies and interventions [16].

Several studies have shown that dietary diversity is positively associated with overall diet quality, micronutrient intake, and better nutritional status of young children and household food security [17-19]. Additionally, high socioeconomic status (SES), as measured by maternal education and employment, may be associated with healthier overall dietary patterns, dietary quality, and adequate dietary diversity in low- and middle-income countries [20-23]. A recent study conducted in India with large-scale data suggested that both individual and contextual socioeconomic factors are associated with diversified dietary intake among children [24]. It is speculated that children in wealthier households grow better for a number of reasons, among which improved nutrient adequacy may be one important mechanism that household wealth and resources translate into better outcomes for children [21]. Wealthier households are expected to have the resources to purchase more food and thus have diverse diets compared to poor households. A higher parental educational level is associated with better employment opportunities and higher incomes, and may translate into higher purchasing power and better nutrition knowledge [21].

In this study, we used the most recent nationally representative data in India to examine the socioeconomic patterning in consumption of specific food items, food groups, and adequacy in dietary diversity among 6-23 months old children.

\section{Methods}

\section{Data}

Data for this analysis were obtained from the recently conducted National Family Health Survey 2015-16 (NFHS-4), which is a cross-sectional, nationally representative survey that provides information on population, health, and nutrition for India and each state/union territory [11]. The NFHS-4, for the first time, provides district-level estimates for many health and nutrition indicators [11]. The details about stratified multistage random sampling procedure employed in the NFHS-4 are provided elsewhere [25].

\section{Study population and sample size}

The NFHS-4 gathered data on all children born within 5 years from the survey year in each household selected to participate. We restricted the main analysis of our study to children aged 6-23 months given that the WHO/UNICEF Infant and Young Child Feeding (IYCF) guidelines were developed to capture mean nutrient adequacy in the context of complementary feeding for this specific age group [26]. A total of 74,132 children were eligible for the study. For complete case analysis, a minimum of 94 to a maximum of 158 children were excluded for missing specific food items. The final analytic sample ranged from 73,852 to 74,038 children depending on the outcome (Fig. 1).

\section{Food consumption and dietary diversity}

In the NFHS-4, mothers were asked to provide a 24-h recall of foods and food groups given to their children. The consumption data were collected on a total of 21 food items that the child had consumed the previous day: juice; tinned powdered/fresh milk; formula milk; fortified baby food; soup/clear broth; other liquids; chicken, duck, or other birds; bread, noodles, other grains; potatoes, cassava, tubers; eggs; pumpkin, carrots, squash; dark green leafy vegetables; mangoes, papayas, Vit A fruits; any other fruits; liver, heart, other organ meat; fish, shellfish; beans, peas, or lentils; cheese, yogurt, other milk products; other solid/ semi-solid food; any other meat; and yogurt.

These food items were grouped into seven food groups following the WHO IYCF guidelines [26, 27]: (1) "grains, roots, and tubers" (comprised of soup/clear broth OR bread, noodles, other grains OR fortified baby food OR potatoes, cassava, tubers); (2) "legumes and nuts" (comprised of beans, peas, or lentils); (3) "dairy products" (comprised of formula milk OR tinned powdered/fresh milk; OR cheese, yogurt, other milk products OR yogurt); (4) "flesh foods" (comprised of liver, heart, other organ meat OR fish, shellfish OR chicken, duck, or other birds); (5) "eggs" (comprised of eggs); (6) "vitamin A rich fruits and vegetables" (comprised of pumpkin, carrots, squash OR dark green leafy vegetables OR mangoes, papayas, Vit A fruits); and (7) "other fruits and vegetables" (comprised of any other fruits).

For each child, a dietary diversity score was constructed by collating information on food consumption, which 
74,132 eligible children aged 6-23 months

\begin{tabular}{|lll|}
\multicolumn{3}{|l}{} \\
\multicolumn{1}{|l}{} & & \\
\hline 21 Food item analysis & N missing & N for analysis \\
\hline Juice & 129 & 74,003 \\
Tinned Powdered/ Fresh milk & 120 & 74,012 \\
Formula milk & 158 & 73,974 \\
Fortified Baby Food & 118 & 74,014 \\
Soup/Clear broth & 133 & 73,999 \\
Other Liquids & 140 & 73,992 \\
Chicken, Duck, or Other Birds & 112 & 74,020 \\
Bread, Noodles, Other Grains & 94 & 74,038 \\
Potatoes, Cassava, Tubers & 120 & 74,012 \\
Eggs & 108 & 74,024 \\
Pumpkin, Carrots, Squash & 111 & 74,021 \\
Dark Green Leafy Vegetables & 113 & 74,019 \\
Mangoes, Papayas, Vit A fruits & 116 & 74,016 \\
Any other Fruits & 120 & 74,012 \\
Liver, heart, other organ meat & 119 & 74,013 \\
Fish, Shellfish & 109 & 74,023 \\
Beans, Peas, or Lentils & 114 & 74,018 \\
Cheese, Yogurt, Other Milk Products & 115 & 74,017 \\
Other Solid/Semi-Solid Food & 157 & 73,975 \\
Any Other Meat & 113 & 74,019 \\
Yogurt & 126 & 74,006 \\
\hline
\end{tabular}

\begin{tabular}{|ll|}
\hline 7 Food group analysis & N for analysis \\
\hline Grains, Roots and Tubers & 74,012 \\
Legumes and Nuts & 74,018 \\
Dairy products & 73,918 \\
Flesh Foods & 73,993 \\
Eggs & 74,024 \\
Vit A rich fruits and vegetables & 74,003 \\
Other fruits and vegetables & 73,852 \\
Vit A rich fruits and vegetables & 74,003 \\
Other fruits and vegetables & 73,852 \\
\hline
\end{tabular}

Fig. 1 Flow diagram showing final sample sizes for analyses on specific food items, food groups, and diversified dietary intake, NFHS-4, India, 2015-16

resulted in an integer ranging from zero to seven (i.e., zero indicated that the child did not consume any of the 21 items and a value of seven indicated that the child was fed at least one food item from each of the seven food groups). A binary variable was constructed from the total dietary diversity score to indicate whether the child diet over the last $24 \mathrm{~h}$ was adequately diverse. Children were considered to have adequately diversified dietary intake (ADDI) if they had food items from at least four of the seven food groups, while a score of 3 or less was considered to be inadequate $[26,27]$.

\section{Main explanatory variables}

The main explanatory variables related to SES included household wealth and maternal education. In the NFHS-4, 
household wealth was calculated from an index of standard household assets and indicators of housing quality through principal component analysis [11, 28, 29]. Each household was assigned a weight score, and the resulting asset scores were standardized according to a normal distribution of zero and a standard deviation of one, and then divided into quintiles based on rank [30]. Maternal education was specified as a categorical variable: no education ( 0 year of education), primary complete (1-5 years of education), secondary complete (6-8 years of education), high school and above ( $9+$ years of education).

\section{Other covariates}

A range of additional covariates were also included as they may influence child dietary diversity and food consumption pattern. These variables included child's age (6-11 months, 12-23 months) and sex, birth order (1, 2, 3, 4+), religion (muslim, hindu, christian, or other/no religion), caste/tribe (scheduled caste, scheduled tribes, other backward class, general), and place of residence (rural, urban).

\section{Statistical analyses}

All analyses were conducted using STATA statistical software package Version 14 (College Station, Texas). Standard descriptive statistics were calculated for all variables, including means and standard deviations. Differences in categorical variables were tested using $\chi^{2}$ tests. We assessed the possibility of multicollinearity between the covariates by examining a correlation matrix of covariates. All pairwise Pearson correlation coefficients were less than 0.5, suggesting that multicollinearity was not a major concern. Due to the non-proportional allocation of the sample to the different survey domains and to their urban and rural areas, we included NFHS-4 sampling weights for our analysis to ensure the actual representativeness of the survey results at the national level and as well as at the domain level [11]. Since NFHS-4 sample is a two-stage stratified cluster sample, sampling weights were calculated based on sampling probabilities separately for each sampling stage and for each cluster [11].

A series of multivariable logistic regression models were used to estimate the odds ratios (ORs) of household wealth status and mother's education on consumption of specific food items, food groups, and ADDI. Model 1 showed the associations between household wealth and maternal education and each outcome after adjusting for child's age and sex only, and Model 2 additionally adjusted for child's birth order, caste, religion, and urban/rural residence. Three sensitivity analyses were performed. First, given that undernutrition persists in older children as well, we assessed the prevalence in consumption of food items and food groups among 43,593 children aged 24-59 months with dietary data. We also assessed the association between SES and ADDI in this age group, with adequate diversity defined as the top two quintiles of the dietary diversity score and inadequate diversity defined as the bottom three quintiles of the dietary diversity score. Second, interaction between household wealth and maternal education was tested in respect to the outcome of ADDI for children aged 623 months. Third, we assessed whether adjusting for states' fixed effects changed the findings from our main analysis.

\section{Ethical considerations}

The study was reviewed by Harvard T.H. Chan School of Public Health Institutional Review Board and was considered exempt from full review because the study was based on an anonymous public use data set with no identifiable information on the study participants.

\section{Results}

Table 1 shows the distribution of our sample by selected characteristics and consumption of specific food items, food groups, and diversified dietary intake. About half of the children in our sample were girls and $38 \%$ were first-order births. Almost half of the mothers had a secondary education while $27 \%$ had no education. Less than a quarter $(24 \%)$ of the children belonged to the poorest wealth quintile households. A majority (79\%) belonged to the Hindu religion, $44 \%$ belonged to other backward class, and $72 \%$ lived in a rural residence. Regarding the prevalence of consumption for each of the 21 food items among children aged 6-23 months, $63 \%$ of the children consumed bread, noodles, other grains, followed by tinned powdered/fresh milk $(40 \%)$, dark green leafy vegetables $(28 \%)$, any other fruits (24\%), other liquids (21\%), potatoes, casavas, tubers (21\%), other solid/semi solid food (21\%), pumpkin, carrots, squash (20\%), juice (20\%), mangoes, papayas, Vit A fruits (18\%), soup/clear broth (16\%), fortified baby food (16\%), and beans, peas, and lentils (13\%) in the previous $24 \mathrm{~h}$ (Table 1). With regards to the seven food groups, the consumption was the highest for grain, roots, and tubers (74\%), followed by dairy products $(55 \%)$, other fruits and vegetables $(37 \%)$, Vit A rich fruits and vegetables (29\%), and the lowest for eggs (14\%), legumes and nuts (13\%), and flesh foods (10\%) in the last $24 \mathrm{~h}$.

A significant difference $(p<0.0001)$ was found in the consumption of juice, fortified baby food, soup/clear broth, formula milk, fortified baby food, mangoes, papayas, Vit A fruits, any other fruits, cheese, yogurt, other milk products by household wealth status among children age 623 months (Table 2). For example, the consumption of juice 
Table 1 Distribution $(N, \%)$ of children by selected characteristics and consumption of specific food items, food groups, and diversified dietary intake in the last $24 \mathrm{~h}$, NFHS-4, India 2015-16

\begin{tabular}{ll}
\hline Selected characteristics & Number \\
\hline
\end{tabular}

Age of child (months)

$$
6-11
$$

$$
\text { 12-23 }
$$

48,820

65.9

Sex of the child

Male

$38,740 \quad 52.3$

Female

$35,298 \quad 47.7$

Birth order

1

2

$28,326 \quad 38.3$

3

$4+$

$24,612 \quad 33.2$

$11,267 \quad 15.2$

$9,833 \quad 13.3$

Mother's education ${ }^{\mathrm{b}}$

No education

Primary complete

20,265

27.4

Secondary complete

High school and above

$10,019 \quad 13.5$

$35,087 \quad 47.4$

$8,667 \quad 11.7$

Household wealth ${ }^{\mathrm{c}}$

Poorest

18,070

Poorer

16,050

15,127

13,719

Richer

11,072

Richest

Religion $^{\mathrm{d}}$

Hinduism

58,265

Muslim

Christian

12,19

1,550

2,032

24.4

Others/No religion

Caste/Tribe $^{\mathrm{e}}$

Scheduled caste

16,108

7,726

21.8

Scheduled tribe

Other backward class

32,593

14,237

3,375

Missing caste

Place of residence

Urban

20,804

Rural

53,233

28.2

71.9

Consumption of specific food items

Juice

$14,427 \quad 19.5$

Tinned powdered/Fresh milk

29,670

40.1

Formula milk

7,699

10.4

Fortified baby food

11,586

$12,137 \quad 16.4$

$15,768 \quad 21.3$

$3,525 \quad 4.8$

$46,549 \quad 62.9$

$15,776 \quad 21.3$

Bread, noodles, other grains

Potatoes, cassava, tubers
Table 1 (continued)

\begin{tabular}{lrl}
\hline Selected characteristics & Number & Percent $^{\mathrm{a}}$ \\
\hline Eggs & 10,651 & 14.4 \\
Pumpkin, carrots, squash & 14,573 & 19.7 \\
Dark green leafy vegetables & 21,045 & 28.4 \\
Mangoes, papayas, Vit A fruits & 13,651 & 18.4 \\
Any other fruits & 17,652 & 23.9 \\
Liver, heart, other organ meat & 3,809 & 5.2 \\
Fish, shellfish & 3,526 & 4.8 \\
Beans, peas, or lentils & 9,825 & 13.3 \\
Cheese, yogurt, other milk products & 6,999 & 9.5 \\
Other solid/Semi-solid food & 15,625 & 21.1 \\
Any other meat & 2,862 & 3.9 \\
Yogurt & 7,007 & 9.5 \\
Specific food groups & & \\
Grains, roots and tubers & 54,420 & 73.5 \\
Legumes and nuts & 9,825 & 13.3 \\
Dairy products & 40,841 & 55.3 \\
Flesh foods & 7,370 & 10.0 \\
Eggs & 10,652 & 14.4 \\
Vit A rich fruits and vegetables & 21,135 & 28.6 \\
Other fruits and vegetables & 27,128 & 36.7 \\
Diversified dietary intake score (mean, & & $2.26(2.24-2.27)$ \\
95\% confidence interval) & & \\
Diversified dietary intake & & \\
Inadequate & 56,740 & 76.8 \\
Adequate & 17,112 & 23.2 \\
\hline
\end{tabular}

${ }^{\text {a}}$ Percent for age, sex, birth order, maternal education, household wealth, religion, caste, and place of residence was calculated based on $N=74,038$; Percent for consumption of specific food item, food group, and diversity dietary intake was calculated based on $N$ of $73,852-74,038$, depending on the outcome (see Fig. 1)

${ }^{\mathrm{b}}$ Education: No education (0 years of education), primary complete: (1-5 years of education), secondary complete (6-8 years of education), high school and above $(9+$ years of education)

${ }^{c}$ Households are given scores based on the number and kinds of consumer goods they own, ranging from a television to a bicycle or car, and housing characteristics such as source of drinking water, toilet facilities, and flooring materials. These scores are derived using principal component analysis. National wealth quintiles are compiled by assigning the household score to each usual (de jure) household member, ranking each person in the household population by their score, and then dividing the distribution into five equal categories, each with $20 \%$ of the population

${ }^{\mathrm{d}}$ Others include Sikh, Buddhist, Jain, Jewish, Zoroastrian

${ }^{\text {e}}$ Scheduled castes and scheduled tribes are identified by the Government of India as socially and economically backward and needing protection from social injustice and exploitation. Other backward class is a diverse collection of intermediate castes that were considered low in the traditional caste hierarchy but are clearly above scheduled castes. General are thus a default residual group that enjoys higher status in the caste hierarchy 


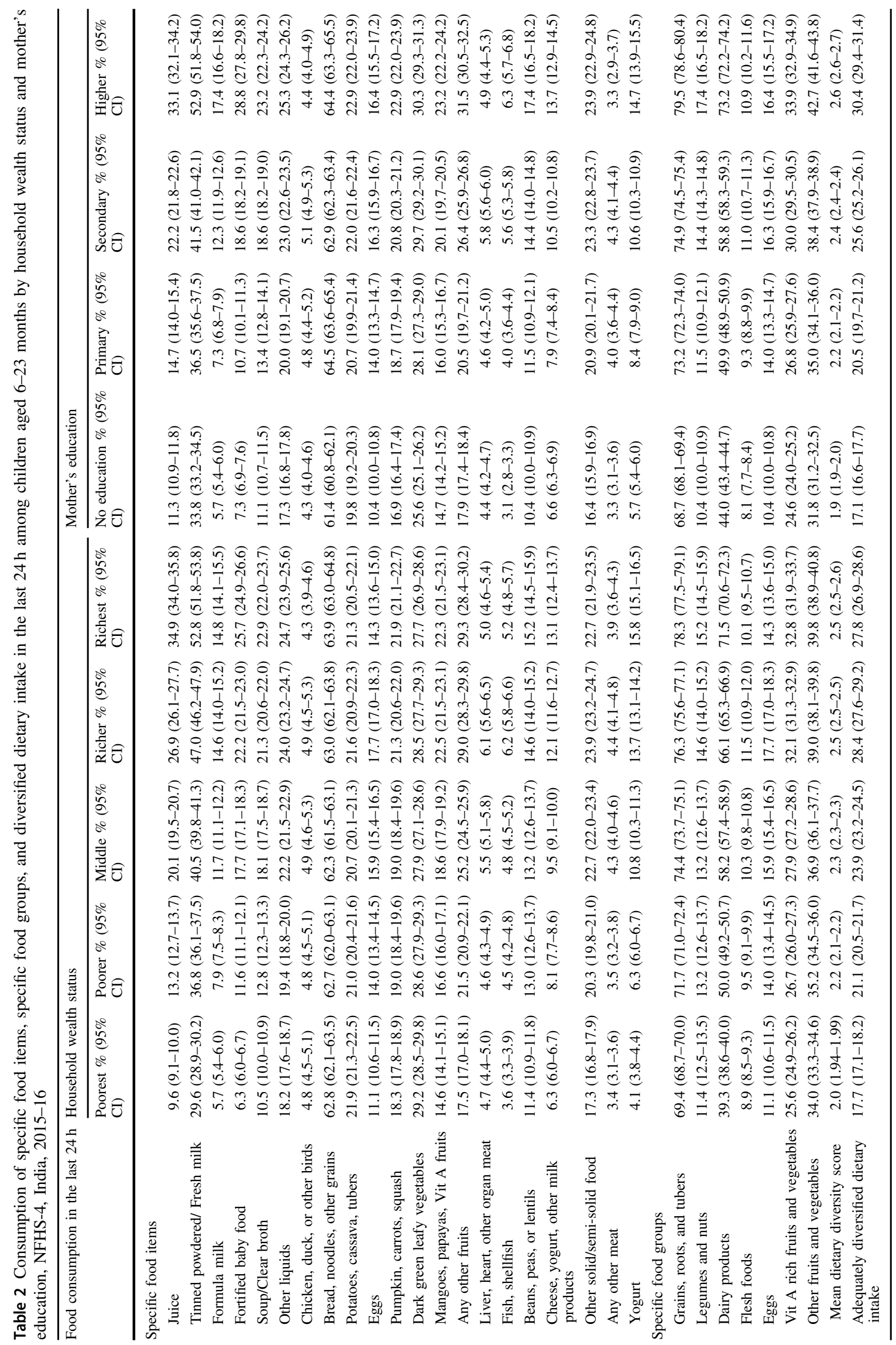


( $10 \%$ vs $35 \%$ ), fortified baby food ( $6 \%$ vs $26 \%$ ), and yogurt (4\% vs $16 \%$ ) was roughly four times greater for the richest households compared to the poorest households. The percentage of children from the richest households that had consumed formula milk ( $6 \%$ vs $15 \%$ ), soup/clear broth (11\% vs $23 \%)$, cheese, yogurt, and other milk products $(6 \%$ vs $13 \%)$ was twice than that in the poorest households. Food group-wise, the largest differential by household wealth was noticed in the consumption of dairy products (39\% vs $72 \%$ ) followed by Vit A rich fruits and vegetables (26\% vs $33 \%$ ) and other fruits and vegetables (34\% vs $40 \%$ ). The mean dietary diversity score ranged from 2.0 among the poorest households to 2.5 among the richest households. A total of $18 \%$ of the children from the poorest households had ADDI compared to $28 \%$ among the richest households.

A substantial differential $(p<0.0001)$ was also found in the consumption of several food items by maternal education among children aged 6-23 months (Table 2). For example, almost four times differential was found in the consumption of fortified baby food (7\% vs $29 \%$ ); three times in the consumption of juice (11\% vs $33 \%$ ), formula milk (6\% vs $17 \%$ ), and yogurt ( $6 \%$ vs $17 \%$ ); twice in soup/ clear broth (11\% vs $23 \%)$, any other fruits ( $18 \%$ vs $32 \%)$, fish and shellfish (3\% vs 6\%), cheese, yogurt, and other milk products ( $7 \%$ vs $14 \%$ ) between children of mothers with no education and those of mothers with high school or above education. Food group-wise, the largest differential by maternal education was found for the consumption of dairy products ( $44 \%$ vs $73 \%$ ), followed by Vit A rich fruits and vegetables ( $25 \%$ vs $34 \%$ ), and other fruits and vegetables ( $32 \%$ vs $43 \%$ ). The mean dietary diversity score and ADDI ranged from 1.9 and $17 \%$ among children of mothers with no education to 2.6 and $30 \%$ among those whose mothers have completed high school education or above, respectively.

Table 3 presents the ORs with 95\% confidence intervals (CIs) of household wealth status on the consumption of 21 specific food items and seven food groups among children aged 6-23 months in two separate models. After adjusting for child's age and sex (Model 1), the odds of consuming fortified baby food, yogurt, juice, and dairy products were found to be four times or more when comparing children from the richest versus the poorest households. Children from the richest households also had higher odds of consuming tinned powdered/fresh milk, formula milk, cheese, yogurt, other milk products, and soup/clear broth. The ORs attenuated in the fully adjusted model (Model 2). Compared to children from the poorest households, those from the richest households were more likely to consume fortified baby food, juice, yogurt, tinned powdered/fresh milk, formula milk, cheese, yogurt other milk product, and mangoes, papayas, Vit A fruits, with ORs ranging from 1.09 to 1.48 . Among the food groups, the consumption of dairy was three times higher among children from the richest as compared to children from the poorest households. Overall, children in the wealthiest households had higher odds of ADDI than children in the poorest households: OR: $1.81(95 \% \mathrm{CI}: 1.71,1.92)$ in Model 1 and OR: 1.28 (95\% CI: 1.18, 1.38) in Model 2 (Fig. 2a).

Table 4 shows the adjusted ORs with $95 \%$ CIs for maternal education on the consumption of 21 specific food items and seven food groups among children aged 623 months in two separate models. After adjusting for child's age and sex (Model 1), the odds of specific food consumption was found to be more than five times higher among children whose mothers had the highest education as compared to children whose mothers had no education for fortified baby food; three times higher for juice and formula milk; and two times higher for tinned powdered fresh milk, soup/clear broth, and cheese, yogurt, other milk products. The association between maternal education and consumption for the following food items remained significant after controlling for household wealth and other covariates in the final adjusted model (Model 2): fortified baby food, formula milk, juice, tinned powdered/fresh milk, beans, peas, lentils, soup/clear broth, cheese, yogurt, other milk products, other liquids, any other fruits, pumpkin, carrots, squash, and potatoes, cassava, tubers. Children of highly educated mothers had significantly higher odds of consuming all of the seven food groups (i.e., ORs ranged from 1.42 (95\% CI: $1.32,1.52)$ for Vit A rich fruits and vegetables to OR: 1.74 (95\% CI: $1.62,1.86$ ) for dairy products). The odds of having ADDI was two times higher (OR: 2.03; 95\% CI: $1.91,2.16$ ) among children of mothers with the highest education versus no education in Model 1 and attenuated slightly in the fully adjusted Model 2 (OR: 1.75; 95\% CI: 1.63, 1.90) (Fig. 2b).

The sensitivity analysis with 24-59 months old children suggested similar patterns of consumption in specific food items and food groups. The mean diversified dietary intake score was lower (1.83) for older children (Supplementary Table 1). Moreover, SES had a weaker association with ADDI among 24-59 months old children (Supplementary Table 2). After adjusting for other covariates, children from the richest wealth quintile had $25 \%$ higher odds of ADDI compared to those from the poorest quintile (OR: 1.25; $95 \%$ CI: $1.04,1.50)$ and children of mothers with the highest education had $47 \%$ higher odds of ADDI compared to the reference group of no maternal education (OR: 1.47; $95 \%$ CI: $1.28,1.67)$. We found no significant interaction between household wealth and maternal education in respect to ADDI among children 6-23 months old $(p=0.175)$. Finally, accounting for states' fixed effect further attenuated the association between household wealth and ADDI (OR 


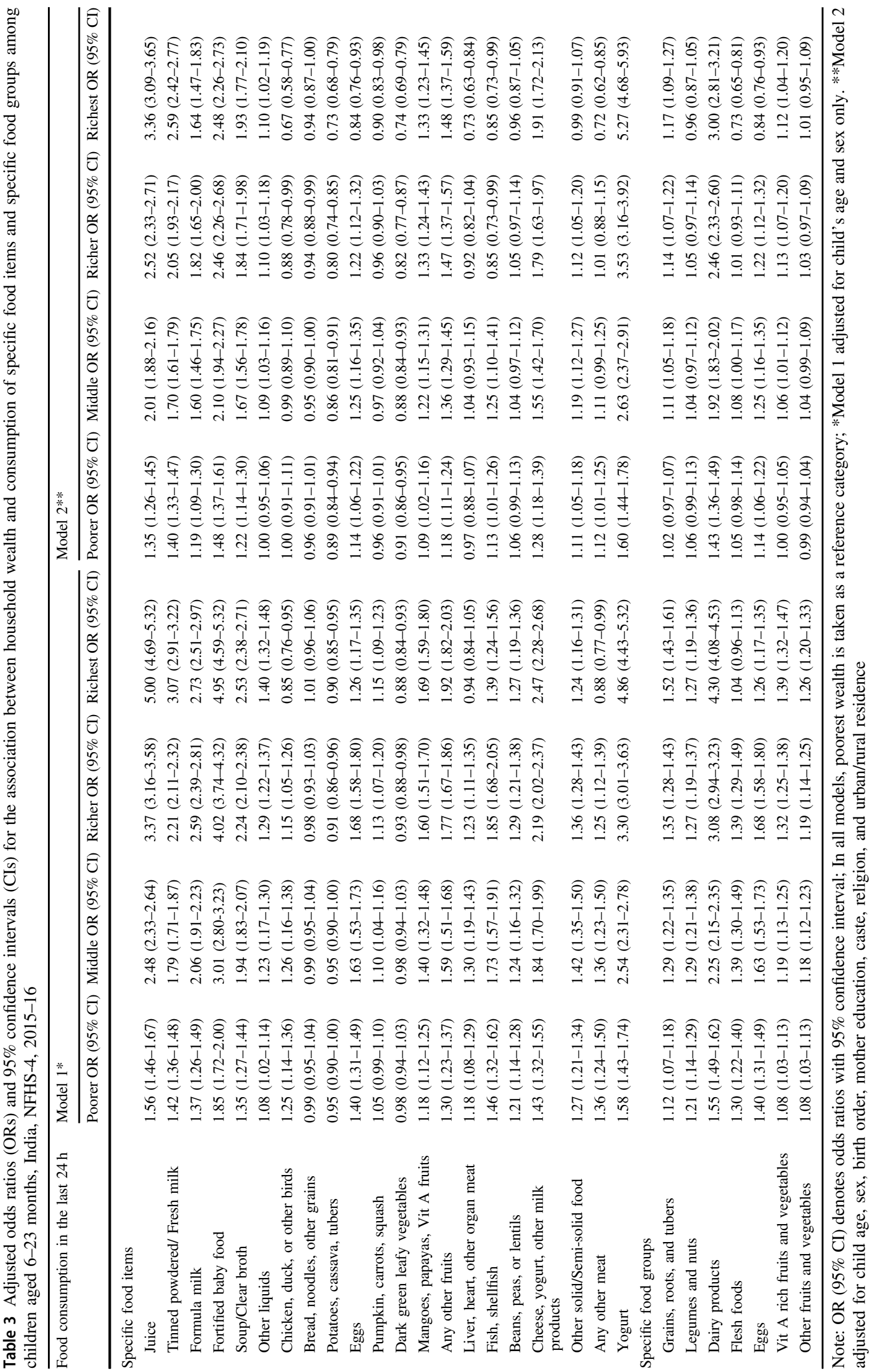


Fig. 2 Adjusted odds ratios (ORs) and $95 \%$ confidence intervals (CIs) for the association between (a) household wealth and (b) mother's education in respect to consumption of adequately diversified dietary among children aged 6-23 months, India, NFHS-4, 2015-16. "Model 1 adjusted for child's age and sex only; ${ }^{\circledR}$ Model 2 adjusted for child age, sex, birth order, caste, religion, urban/rural residence, and maternal education for Fig. 2a and household wealth for Fig. $2 b$

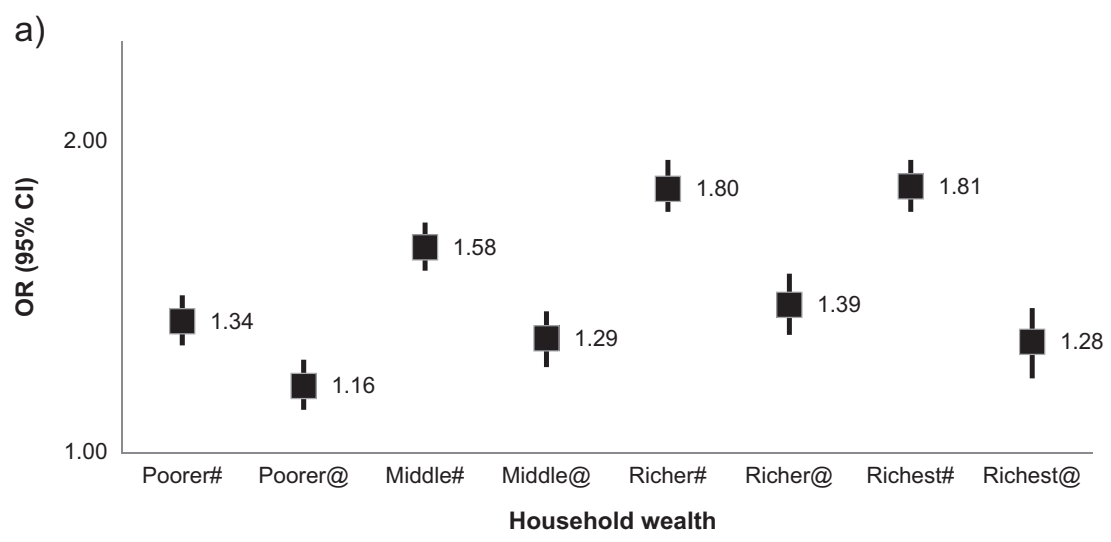

b)

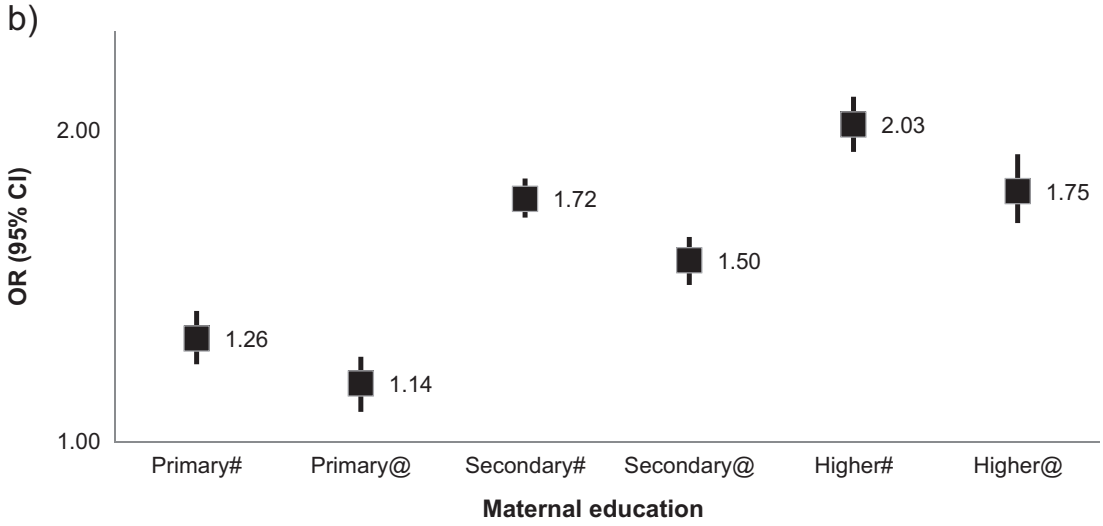

comparing the highest vs lowest wealth quintile: 1.53 ; $95 \%$ CI: $1.40,1.67)$ as well as the association between maternal education and ADDI (OR comparing the highest vs no maternal education: 1.32 ; 95\% CI: $1.22,1.44$ ) (Supplementary Table 3).

\section{Discussion}

In this study, we used the latest nationally representative cross-sectional data from India to assess food consumption pattern and ADDI among children aged 6-23 months. We found that SES, as measured by household wealth and maternal education, was associated with ADDI among children. While maternal education was more strongly associated with consumption of essential food items and all food groups, household wealth status was significantly associated with consumption of dairy group only. In general our findings are consistent with results from past [21, 23, $24,31]$ and recent studies [32] conducted in LMICs and India, which found a positive relationship between measures of individual-level socioeconomic status and specific food item/food group consumption and diversified dietary intake.
More specifically, we found that consumption of food items, such as pumpkin, carrots, squash, dark green leafy vegetables, liver, heart, other organ meat, fish, shellfish, legumes and nuts, and flesh food, was significantly associated with maternal education but not with household wealth. Thus, maternal education may be more important than wealth in determining the intake of more variety of food items and food groups, although both measures of SES had similar influence on the overall ADDI. The consumption of food items that are relatively cheap in India (i.e., pumpkins, carrots, dark green leafy vegetables) can be further emphasized to improve diet diversity at the population level.

While dietary diversity score generally improved with higher maternal education and household wealth, the consumption of packaged food products also increased for the higher SES groups. For instance, the consumption of juice was higher for richer vs poorer households (35\% vs $10 \%)$ and by maternal education ( $33 \%$ vs $11 \%$ ). At the same time, there was not much difference in the consumption of healthy items (i.e., green leafy vegetables/pulses etc.) by the SES groups. Further investigation of the trend in consumption of packaged food and other nutritious fresh food options by SES may be informative. 


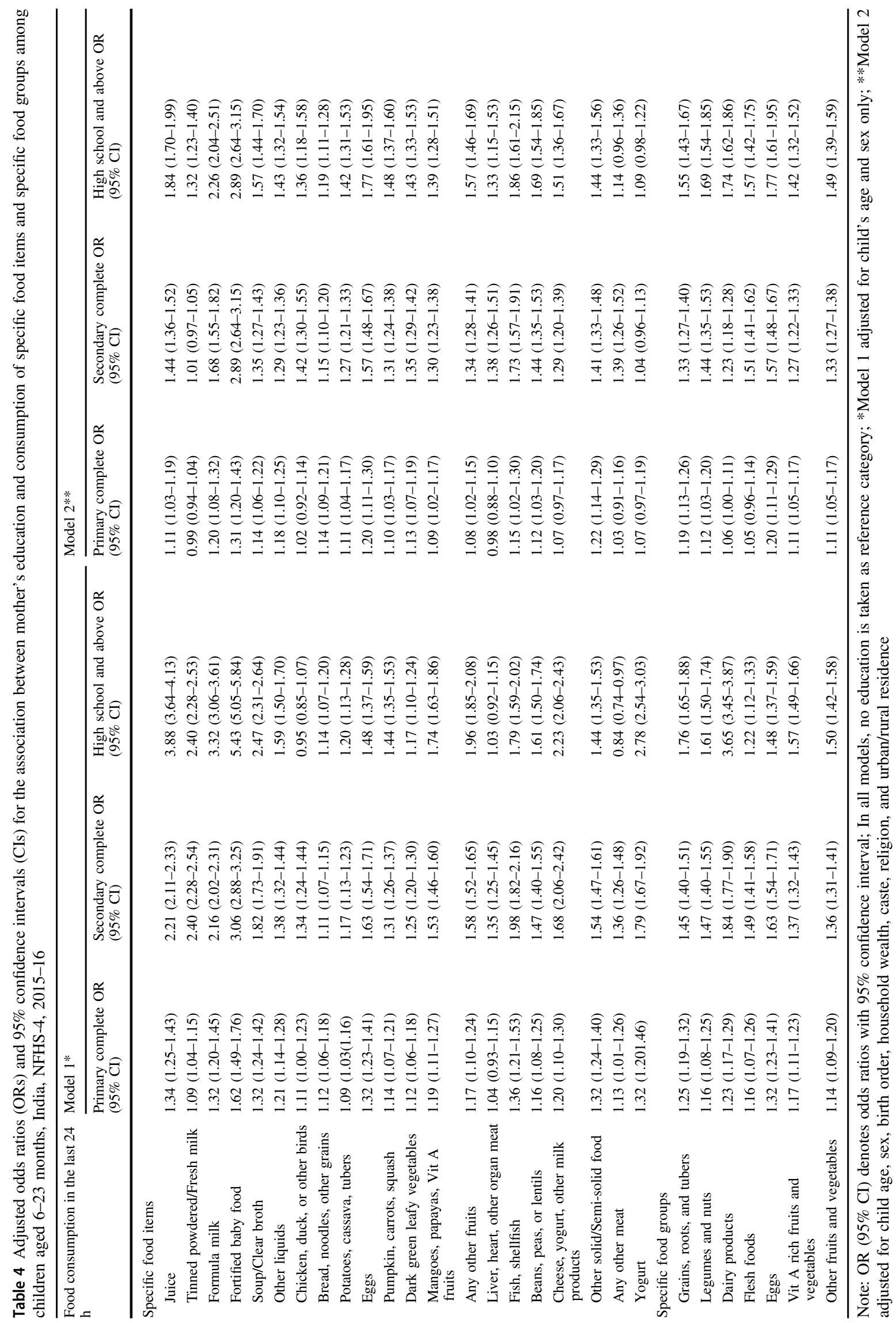




\section{Limitations of the study}

There are several limitations to this study. First, the study sample may be subject to survival bias. Children who were not alive at the time of the survey were excluded from the sample. Such children may be more likely to represent the most disadvantaged population segments and have the poorest dietary diversity score given the relationship between socioeconomic disadvantage, dietary intake, and child health. Second, although 24-h maternal recall of dietary intake is the measurement technique recommended by the WHO to assess child dietary intake, the measurement relies on self-report and therefore may be subject to recall error [33]. Although such error may result in some misclassification, the reliance on a $24-\mathrm{h}$ recall period is more accurate than other measurement techniques that rely on longer reference periods [34]. Third, causality cannot be assessed as this study used observational methods and cross-sectional data. Finally, the NFHS-4 included information on only a few unhealthy food items such as biscuits etc. and other fried packaged food items normally consumed by Indian children.

\section{Conclusion}

In this study we observed relatively shallow SES gradient in children's food consumption. While both household wealth and maternal education significantly improved the overall dietary diversity, the strength of the associations was considerably weaker when compared to the SES gradient observed for child anthropometric failures [5, 6]. Furthermore, the associations between SES and consumption of individual food items and food groups were less consistent. Higher maternal education was relatively more strongly associated with consumption of essential food items and all food groups, but household wealth was found to have significant influence on intake of dairy group only. The increase in the consumption of packaged food among higher SES groups was also alarming. This implies that caregiver's general education level alone is not sufficient to make healthy feeding choices for the children. Instead, exposure to accurate information related to diet and nutrients may be important given the current nutritional transition in India. Interventions designed to improve food consumption and diversified dietary intake among Indian children need to be more universal given the overall high prevalence of inadequate dietary diversity (77\%) and relatively small differentials by SES.

Acknowledgments The authors acknowledge the support of International Institute for Population Sciences and The DHS Program at ICF (www.dhsprogram.com) for providing access to the 2015-16 Indian National Family Health Survey data.

Funding: The authors have no support or funding to report.

Author contributions SA contributed to conceptualization and design of the study, led the analysis and interpretation of data, and drafted the initial manuscript. RK, JG, and WJ contributed to conceptualization and design of the study, contributed to analysis and interpretation of data, and reviewed the manuscript for important intellectual content. SS and RS contributed to interpretation of findings and reviewed the manuscript for important intellectual content. SVS conceptualized and designed the study, contributed to analysis and interpretation of data, and reviewed the manuscript for important intellectual content. WJ and SVS provided overall supervision.

\section{Compliance with ethical standards}

Conflict of interest The authors declare that they have no conflict of interest.

Publisher's note: Springer Nature remains neutral with regard to jurisdictional claims in published maps and institutional affiliations.

Open Access This article is licensed under a Creative Commons Attribution 4.0 International License, which permits use, sharing, adaptation, distribution and reproduction in any medium or format, as long as you give appropriate credit to the original author(s) and the source, provide a link to the Creative Commons license, and indicate if changes were made. The images or other third party material in this article are included in the article's Creative Commons license, unless indicated otherwise in a credit line to the material. If material is not included in the article's Creative Commons license and your intended use is not permitted by statutory regulation or exceeds the permitted use, you will need to obtain permission directly from the copyright holder. To view a copy of this license, visit http://creativecommons. org/licenses/by/4.0/.

\section{References}

1. Ruel MT, Arimond M. Dietary diversity and growth: an analysis of recent demographic and health surveys. In: Brower ED, Traore AS, Treche S, editors. Food based approaches for a healthy nutrition in West Africa. Ouagadougou: University Press; 2004.

2. Charmarbagwala R, Ranger M, Waddington H, White $H$. The determinants of child health and nutrition: a meta-analysis. Washington, DC: World Bank; 2004.

3. Khan REA, Raza MA. Nutritional status of children in Bangladesh: measuring Composite Index of Anthropometric Failure $(C I A F)$ and its determinants. Published. Pak J Commer Social Sci. 2014;8:11-23.

4. McDonald CM, McLean J, Kroeun H, Talukder A, Lynd LD, Green TJ. Household food insecurity and dietary diversity as correlates of maternal and child under nutrition in rural Cambodia. Eur J Clin Nutr. 2015;69:242-6.

5. Corsi DJ, Mejía-Guevara I, Subramanian SV. Improving household-level nutrition-specific and nutrition-sensitive conditions key to reducing child undernutrition in India. Soc Sci Med. 2016;157:189.

6. Kim R, Mejía-Guevara I, Corsi DJ, Aguayo VM, Subramanian SV. Relative importance of 13 correlates of child stunting in South Asia: Insights from nationally representative data from 
Afghanistan, Bangladesh, India, Nepal, and Pakistan. Soc Sci Med. 2017;187:144-54.

7. Emdadul Hauqe S, Sakisaka K, Rahman M. Examining the relationship between socioeconomic status and the double burden of maternal over and child under-nutrition in Bangladesh. Eur J Clin Nutr. 2018. https://doi.org/10.1038/s41430-018-0162-6.

8. Balarajan Y, Selvaraj S, Subramanian SV. Health care and equity in India. Lancet. 2011;377:505-15.

9. Kehoe SH, Krishnaveni GV, Veena SR, Guntupalli AM, Margetts $\mathrm{BM}$, Fall $\mathrm{CH}$, et al. Diet patterns are associated with demographic factors and nutritional status in South Indian children. Matern Child Nutr. 2014;10:145-58.

10. Aurino E. Do boys eat better than girls in India? Longitudinal evidence on dietary diversity and food consumption disparities among children and adolescents. Econ Hum Biol. 2016. https:// doi.org/10.1038/s41430-018-0162-6.

11. International Institute for Population Sciences (IIPS) and ICF. National Family Health Survey (NFHS-4), 2015-16. Mumbai: IIPS; 2017.

12. Pingali P. In: Pingali P, Evenson R, editors. Handbook of agricultural economics. Amsterdam: Elsevier; 2010. p.3867-94.

13. Grebmer VK, Bernstein J, Hossain N, Brown T, Prasai N, Yohannes Y, et al. Global Hunger Index: The inequalities of hunger: Synopsis. Washington, DC, Bonn, and Dublin: International Food Policy Research Institute, Welthungerhilfe, and Concern Worldwide; 2017.

14. Ministry of Health and Population (MOHP), New ERA. ICF International Nepal demographic and health survey 2011, Ministry of Health and Population, Kathmandu, Nepal. Calverton : New ERA, and ICF International; 2012.

15. Press Information Bureau, Government of India, Ministry of Women and Child Development. National Nutrition Mission. 2018 17:03 IST. https://www.icds-wcd.nic.in/nnm/home.htm

16. Press Information Bureau, Government of India, Ministry of Women and Child Development. National Nutrition Mission to improve the nutritional indicators of women and children. http:// pib.nic.in/newsite/PrintRelease.aspx?relid=176441

17. Sawadogo PS, Martin-Prevel Y, Savy M, Kameli Y, Traissac P, Traore AS, et al. An infant and child feeding index is associated withthe nutritional status of 6- to 23-month-old children in rural Burkina Faso. J Nutr. 2006;136:656-63.

18. Steyn N, Nel J, Nantel G, Kennedy G, Labadarios D. Food variety and dietary diversity scores in children: are they good indicators of dietary adequacy? Public Health Nutr. 2006;9:644-50.

19. Kennedy GL, Pedro MR, Seghieri C, Nantel G, Brouwer I. Dietary diversity score is a useful indicator of micronutrient intake in non-breast-feeding Filipino children. J Nutr. 2007;137:472-7.

20. Nguyen PH, Avula R, Ruel MT, Saha KK, Ali D, Tran LM, et al. Maternal and child dietary diversity are associated in Bangladesh, Vietnam, and Ethiopia. J Nutr. 2013;143:1176-83.
21. Codjoe SNA, Okutu D, Abu M. Urban household characteristics and dietary diversity: an analysis of food security in Accra, Ghana. Food Nutr Bull. 2016; 37:202-18.

22. Mayén A-L, Marques-Vidal P, Paccaud F, Bovet P, Stringhini S. Socioeconomic determinants of dietary patterns in low- and middle-income countries: a systematic review. Am J Clin Nutr. 2014; 100:1520-31.

23. Harris-Fry H, Azad K, Kuddus A, Shaha S, Nahar B, Hossen M, et al. Socio-economic determinants of household food security and women's dietary diversity in rural Bangladesh: a crosssectional study. J Health Popul Nutr. 2015;33:1.

24. Gausman J, Perkins JM, Lee H-Y, Mejia-Guevara I, Nam Y-S, Lee J-K, et al. Ecological and social patterns of child dietary diversity in India: a population-based study. Nutrition. 2018;53:77-84.

25. International Institute for Population Sciences - IIPS/India and ICF. 2017. India National Family Health Survey NFHS-4 201516. Mumbai, India: IIPS and ICF. http://dhsprogram.com/pubs/ pdf/FR339/FR339.pdf.

26. WHO, UNICEF, USAID, FANTA, AED, UC DAVIS. et al. Indicators for assessing infant and young child feeding practices, Part 2: measurement. Geneva: The World Health Organization; 2010.

27. World Health Organization. Indicators for assessing infant and young child feeding practices part 1 definitions. Washington, DC: World Health Organization, Dept. of Child and Adolescent Health and Development; 2007.

28. Filmer D, Pritchett LH. Estimating wealth effects without expenditure data-or tears: an application to educational enrollments in states of India. Demography. 2001;38:115-32.

29. Howe LD, Hargreaves JR, Huttly SRA. Issues in the construction of wealth indices for the measurement of socio-economic position in low-income countries. Emerg Themes Epidemiol. 2008;5:3.

30. Gwatkin D, Rutstein S, Johnson K, Pande R, Wagstaff A. Socioeconomic differences in health, nutrition and poverty. Washington, DC: World Bank: HNP/Poverty Thematic Group, World Bank; 2000.

31. Torheim L, Ouattara F, Diarra M, Thiam F, Barikmo I, Hatløy A, et al. Nutrient adequacy and dietary diversity in rural Mali: association and determinants. Eur J Clin Nutr. 2004;58:594-604.

32. Nguyen PH, Avula R, Headey D, Tran LM, Ruel MT, Menon P. Progress and inequalities in infant and young child feeding practices in India between 2006 and 2016. Matern Child Nutr. 2018;14:e12663.

33. World Health Organization. Indicators for assessing infant and young child feeding practices: Part 2: Measurement. 2010.

34. Swindale A, Bilinsky P. Household dietary diversity score (HDDS) for measurement of household food access: indicator guide. Washington, DC: Food and Nutrition Technical Assistance Project, Academy for Educational Development; 2006. 